\section{(2) OPEN ACCESS}

\title{
Cardiac biomarkers predict mortality in emergency patients presenting with atrial fibrillation
}

\author{
Jan Niederdöckl, ${ }^{1}$ Alexander Simon, ${ }^{1}$ Sebastian Schnaubelt, ${ }^{1}$ Nikola Schuetz, ${ }^{1}$ \\ Roberta Laggner, ${ }^{2}$ Patrick Sulzgruber, ${ }^{3}$ Alexander 0 Spiel, ${ }^{1}$ Harald Herkner, ${ }^{1}$ \\ Anton N Laggner, ${ }^{1}$ Hans Domanovits ${ }^{1}$
}

'Department of Emergency Medicine, Medical University of Vienna, Vienna, Austria ${ }^{2}$ Department of Trauma Surgery, Medical University of Vienna, Vienna, Austria

${ }^{3}$ Department of Cardiology, Medical University of Vienna, Vienna, Austria

\section{Correspondence to} Dr Jan Niederdöckl, Medizinische Universitat Wien Universitatsklinik fur Notfallmedizin, Wien 1090, Austria; jan_niederdoeckl@ gmx.at

Received 9 February 2018 Revised 21 August 2018 Accepted 5 September 2018 Published Online First 10 November 2018
ABSTRACT

Objectives To assess the predictive value of $\mathrm{N}$-terminal pro-brain natriuretic peptide (NT-proBNP) and highsensitive troponin T (hs-TnT) serum levels for mid-term mortality in patients presenting with symptomatic atrial fibrillation (AF) to an emergency department.

Methods Non-interventional cohort/follow-up study, including consecutive patients presenting to a tertiary care university emergency department due to symptomatic AF between 2012 and 2016. Multivariable Cox proportional hazard regression models were used to estimate the mortality rates and hazards per 100 patientyears (pry) for NT-proBNP and hs-TnT serum levels in quintiles.

Results 2574 episodes of 1754 patients (age 68 (IQR 58-75) years, female gender 1199 (44\%), CHA, DS,VASc 3 (IQR 1-4)) were recorded. Following the exclusion of incomplete datasets, 1780 episodes were available for analysis. 162 patients deceased during the mid-term follow-up (median 23 (IQR 4-38) months); the mortality rate was $4.72 / 100$ pry. Hazard for death increased with every quintile of NT-proBNP by 1.53 (HR; $95 \% \mathrm{Cl} 1.27$ to $1.83 ; p<0.001)$ and by 1.31 (HR; $95 \% \mathrm{Cl} 1.10$ to $1.55 ; p=0.002$ ) with every quintile of hs-TnT in multivariate Cox-regression analysis. No interaction between NT-proBNP and hs-TnT levels could be observed.

Conclusion Elevated NT-proBNP and hs-TnT levels are independently associated with increased midterm mortality in patients presenting to an emergency department due to symptomatic AF.

Trial registration number NCT03272620; Results.

\section{INTRODUCTION}

Atrial fibrillation (AF) as the primary symptom occurs in $3.3 \%$ to $10.0 \%$ of emergency department (ED) admissions. ${ }^{1}$ As to the ongoing ED crowding worldwide, an effective management for patients with symptomatic AF is mandatory. ${ }^{2}{ }^{3}$ Ideally, optimisation of patient management and resource allocation should be based on a time and costeffective risk stratification. ${ }^{4} \mathrm{Up}$ to now, the prognosis prediction is merely derived from established cardiovascular risk scores and thromboembolic risk prediction tools as the $\mathrm{CHA}_{2} \mathrm{DS}_{2}-\mathrm{VAS}$ c score; a biomarker based approach might be then helpful for identification of patients at increased risk for mortality during mid-term follow-up. ${ }^{3}$

Brain natriuretic peptide (BNP) and N-terminal pro-brain natriuretic peptide (NT-proBNP) are established prognostic markers for outcome and mortality in various fields of cardiovascular (CV) disease. ${ }^{5-8}$ Cardiac troponins are sensitive biomarkers for cardiac damage and already slight elevations are associated with adverse outcome in CV disease. ${ }^{9-11}$

It was recently proposed that the combination of a biomarker- approach might improve existing risk stratification tools in patients with $\mathrm{AF}^{81213}$ The aim of the present study was then to evaluate the predictive value of both NT-proBNP and high-sensitive troponin $\mathrm{T}$ (hs-TnT) to predict mid-term mortality in symptomatic AF patients presenting to an ED.

\section{METHODS \\ Design/Setting}

In this single-centre cohort study, consecutive adult patients presenting with $\mathrm{AF}$ at the ED of the Medical University of Vienna from 2012 to 2016 were eligible for inclusion.

\section{Data acquisition}

Following written consent, demographic data, current prescriptions, relevant comorbidities and type of AF according to the current guidelines, duration and AF symptoms were recorded. As guideline definitions for paroxysmal and persistent AF evolved during the study period, both classes have been merged for analysis.

\section{Laboratory values and measurements}

Serum NT-proBNP and hs-TnT assays were processed on Cobas E602-Module (ECLIA, Roche Diagnostics GmbH, Mannheim, Germany) console with a coefficient of variation and the reference range of $5.7 \%$ and $0-14 \mathrm{ng} / \mathrm{L}$ for hs-TnT and 3.7\% and $0-125 \mathrm{pg} / \mathrm{mL}$ for NT-proBNP. The limit of blank was $3 \mathrm{ng} / \mathrm{L}$ for hs-TnT, the limits of detection $5 \mathrm{ng} / \mathrm{L}$ for hs-TnT and $5 \mathrm{pg} / \mathrm{mL}$ for NT-proBNP (according to the CLSI EP17-A guideline).

\section{Mortality data}

Official 'all- cause death' data were provided by the Austrian death registry maintained by the national central statistical office (Statistik Austria, Guglgasse 13, A-1110 Vienna).

\section{Statistics}

We present continuous data as median and 25\% to $75 \% \mathrm{IQR}$, categorised data as absolute count 
Table 1 Association of cardiac biomarkers and mortality

\begin{tabular}{|c|c|c|c|c|c|c|}
\hline & \multicolumn{2}{|c|}{ NT-proBNP } & \multirow[b]{2}{*}{$P$ values } & \multicolumn{2}{|c|}{ hs-TnT } & \multirow[b]{2}{*}{$P$ values } \\
\hline & HR & $95 \% \mathrm{Cl}$ & & HR & $95 \% \mathrm{Cl}$ & \\
\hline \multicolumn{7}{|l|}{ Model } \\
\hline Unadjusted & $1.97 \dagger$ & 1.71 to 2.26 & $<0.001$ & $1.99 \dagger$ & 1.70 to 2.33 & $<0.001$ \\
\hline Adjusted for clinical factors* & $1.53+$ & 1.27 to 1.83 & $<0.001$ & $1.31 \dagger$ & 1.10 to 1.55 & 0.002 \\
\hline Adjusted for $\mathrm{CHA}_{2} \mathrm{DS}_{2}$-VASc & $1.70 \dagger$ & 1.43 to 2.03 & $<0.001$ & $1.52 \dagger$ & 1.28 to 1.81 & $<0.001$ \\
\hline \multicolumn{7}{|l|}{ Sensitivity analysis } \\
\hline First episode only & $1.68 \dagger$ & 1.39 to 2.04 & $<0.001$ & $1.91 \dagger$ & 1.54 to 2.36 & $<0.001$ \\
\hline Random effects models & $2.34 \dagger$ & 1.82 to 3.01 & $<0.001$ & $2.98 t$ & 2.19 to 4.03 & $<0.001$ \\
\hline Continuous scale $\ddagger$ & 1.66 & 1.38 to 2.00 & $<0.001$ & 1.26 & 1.07 to 1.49 & 0.006 \\
\hline
\end{tabular}

*age, female gender, heart failure, chronic obstructive pulmonary disease, serum creatinine, arterial hypertension, coronary artery disease, hyperlipidaemia, diabetes mellitus, peripheral artery disease, history of stroke, history of transient ischemic attack, current smoking, pulmonary vein isolation, beta-blocker and diuretic therapy. tHR for increase of biomarker quintile.

$\ddagger$ After natural log transformation.

and relative frequency (percentage, \%). The entry time for the calculation of the observation period in patient-years (pry) was the first admission with AF at the study centre. Observation time was censored at the date of death or end of the follow-up. For the calculation of the prognostic value of NT-proBNP and hs-TnT, AF episodes with incomplete (only one or no biomarker value at the time of admission) cardiac biomarker information were excluded. To achieve appropriately sized groups including a centrally positioned one, we categorised NT-proBNP $\left(\mathrm{Q}_{\mathrm{bnp}}\right)$ and hs-TnT $\left(\mathrm{Q}_{\mathrm{tnt}}\right)$ into quintiles. To compare baseline data between NT-proBNP and hs-TnT quintiles, we used a $\chi^{2}$ test for categorised variables. We used the Kruskal-Wallis test for continuous data to test the null hypothesis of no difference because the normality assumption was not met for all baseline data. The unit of analysis of our cohort were admissions (episodes) for AF and included $20 \%$ patients, who presented with more than one AF episode. We performed our analysis at the level of episodes, allowing for correlation within patients with multiple episodes using variance component estimates for clustered observations. We calculated mortality rates per 100 pry for NT-proBNP and hs-TnT quintiles and their combinations. We used Cox proportional hazards regression to estimate the hazards of death with NT-proBNP and hs-TnT quintiles simultaneously as the main covariates. We extended the models to assess the independent association of NT-proBNP and hs-TnT with other covariates (table 1).

We also calculated an alternative model with $\mathrm{CHA}_{2} \mathrm{DS}_{2}$-VASc as the summary integrative covariate of clinically relevant factors

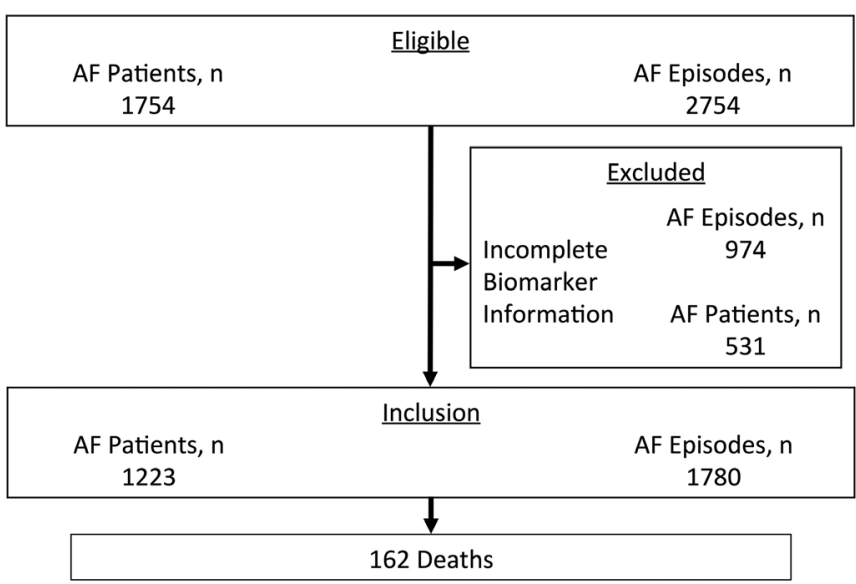

Figure 1 Flow chart of patient inclusion. $A F$, atrial fibrillation. but did not model this covariable with the other factors to avoid multicollinearity. We used the Akaike information criterion for model comparison. We assessed first-order interactions of biomarkers by including interaction terms into the models and tested for deviation from linearity. We performed sensitivity analyses to assess the robustness of our multiepisode approach using the first episode only (ignoring other episodes of the same patients) or using random effects models. To assess the robustness of biomarker categorisation, we modelled biomarkers as covariables on a continuous scale after natural log transformation. We assumed that missing data were missing at random. Given the sample size, we decided not to use any methods of data imputation or replacement.

For data management and analysis, we used MS Excel and Stata 14 for Mac. Generally, a two-sided p value $<0.05$ was considered statistically significant.

\section{RESULTS}

\section{Baseline characteristics}

Patients and episodes

In total 2574 episodes of 1754 patients (age 68 (IQR 58-75) years, female gender 1199 (44\%), $\mathrm{CHA}_{2} \mathrm{DS}_{2}$-VASc 3 (IQR 1-4)) with symptomatic AF were available for analysis (figure 1). Following exclusion of AF episodes with incomplete biomarker datasets, final analysis comprised 1780 episodes with both NT-proBNP and hs-TnT levels.

Median heart rate was at 122 (IQR 103-140) bpm. Paroxysmal/persistent $\mathrm{AF}$ was the most frequent type of $\mathrm{AF}$ in all biomarker subgroups. With increasing levels of NT-proBNP and hs-TnT, the hazard for permanent AF increased. The median duration of current AF episodes was 2 (IQR 1-4) and 3 (IQR 2-10) in the lowest, 20 (IQR 8-48) and 8 (IQR 3-48) hours in the highest respective NT-proBNP and hs-TnT quintiles. Duration of current AF correlated directly with biomarker levels. Cardiovascular risk factors were more prevalent in patients with higher biomarker levels (tables 2 and 3).

The total observed time was 3433 pry with a median follow-up duration of 23 (IQR 4-38) months. A total of 162 patients deceased during mid-term follow-up; mortality rate was 4.72 per 100 pry. Mortality rate increased linearly by biomarker levels $(\mathrm{p}<0.001)$.

\section{NT-proBNP, hs-TnT and mortality}

We could observe a significant crude association of mortality with both increasing NT-proBNP quintiles and increasing 
는

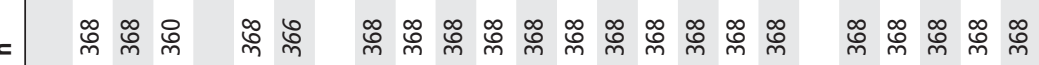

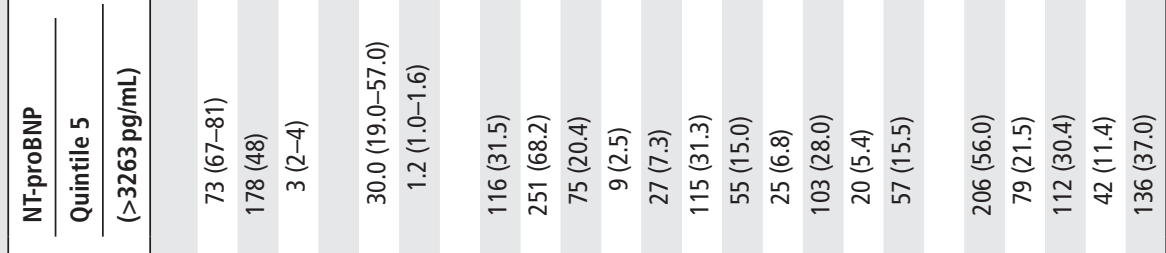

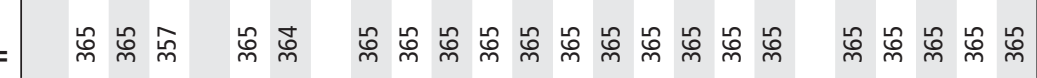

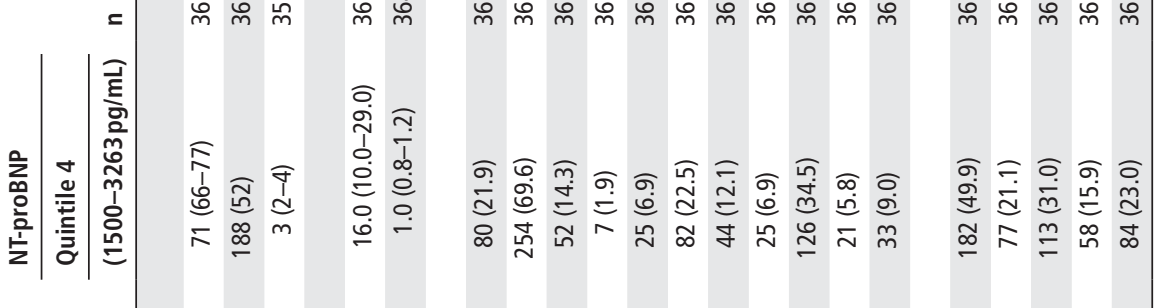

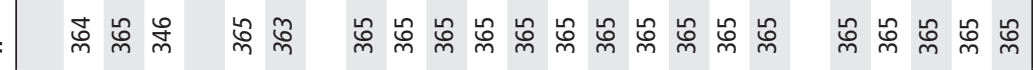

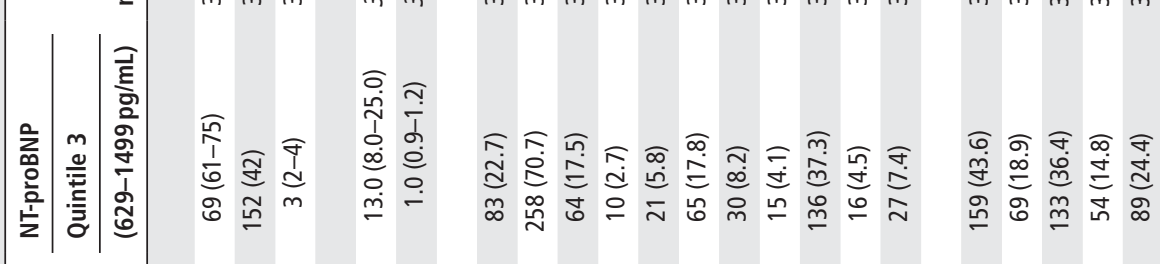

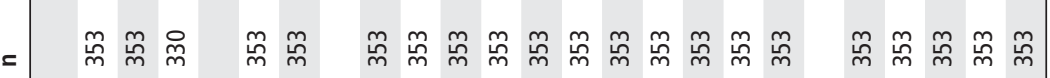

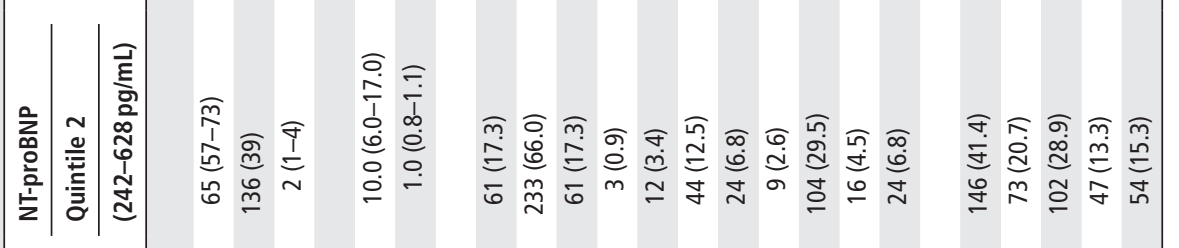

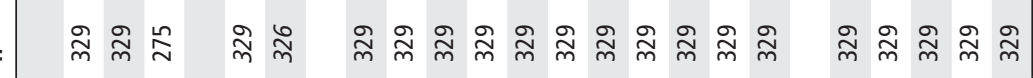

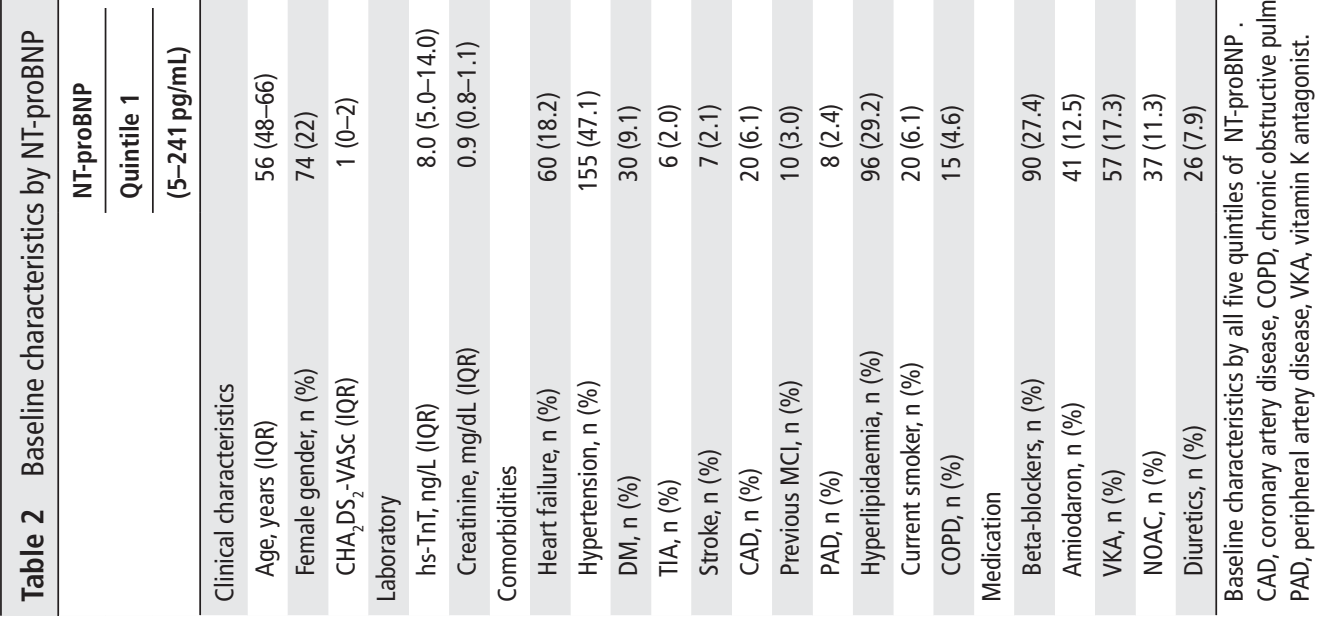




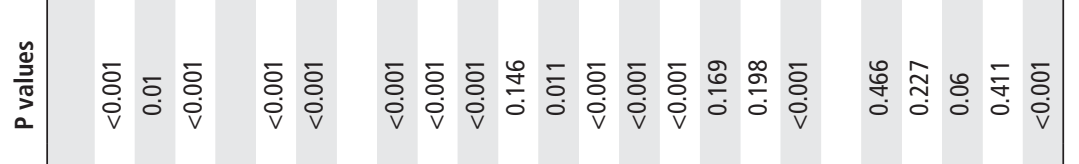

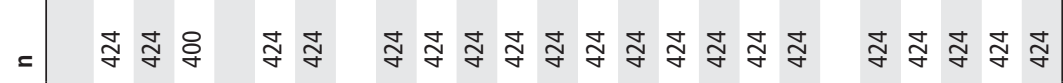

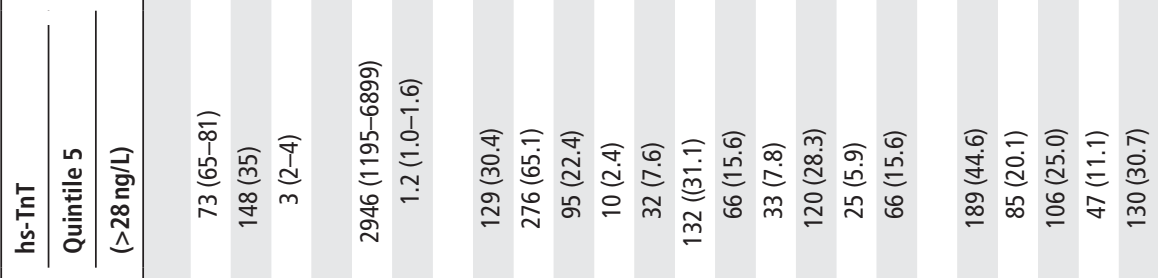

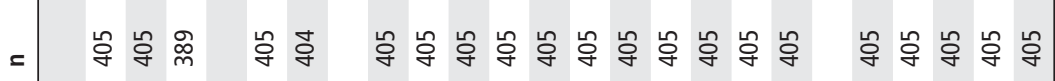

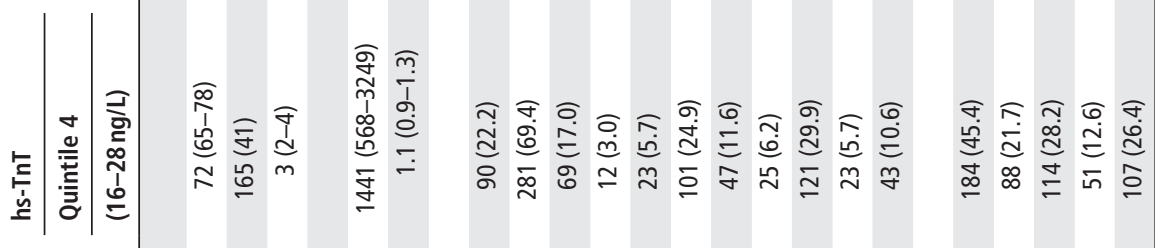

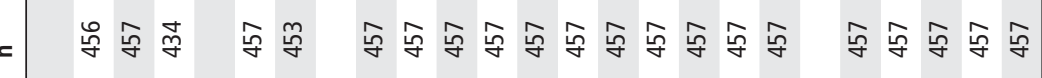

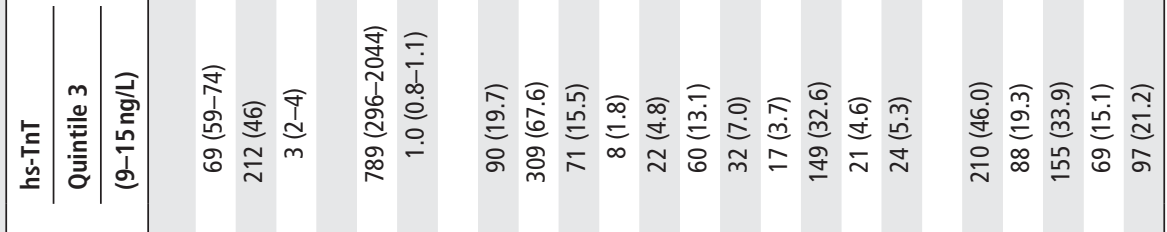

$=$ 志志

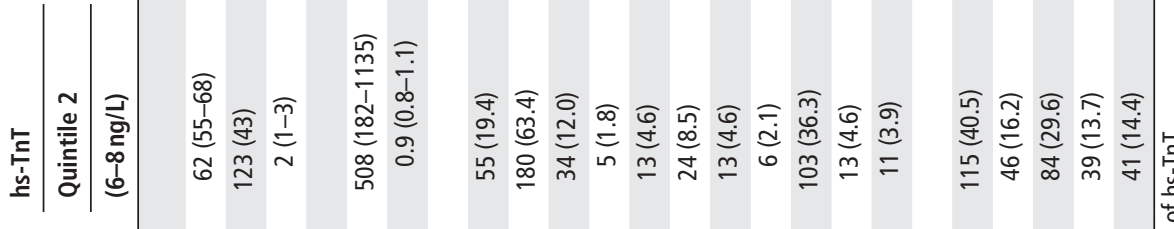

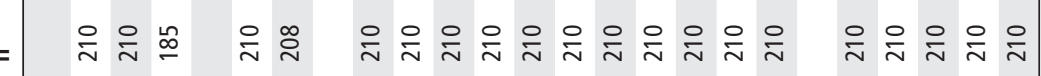

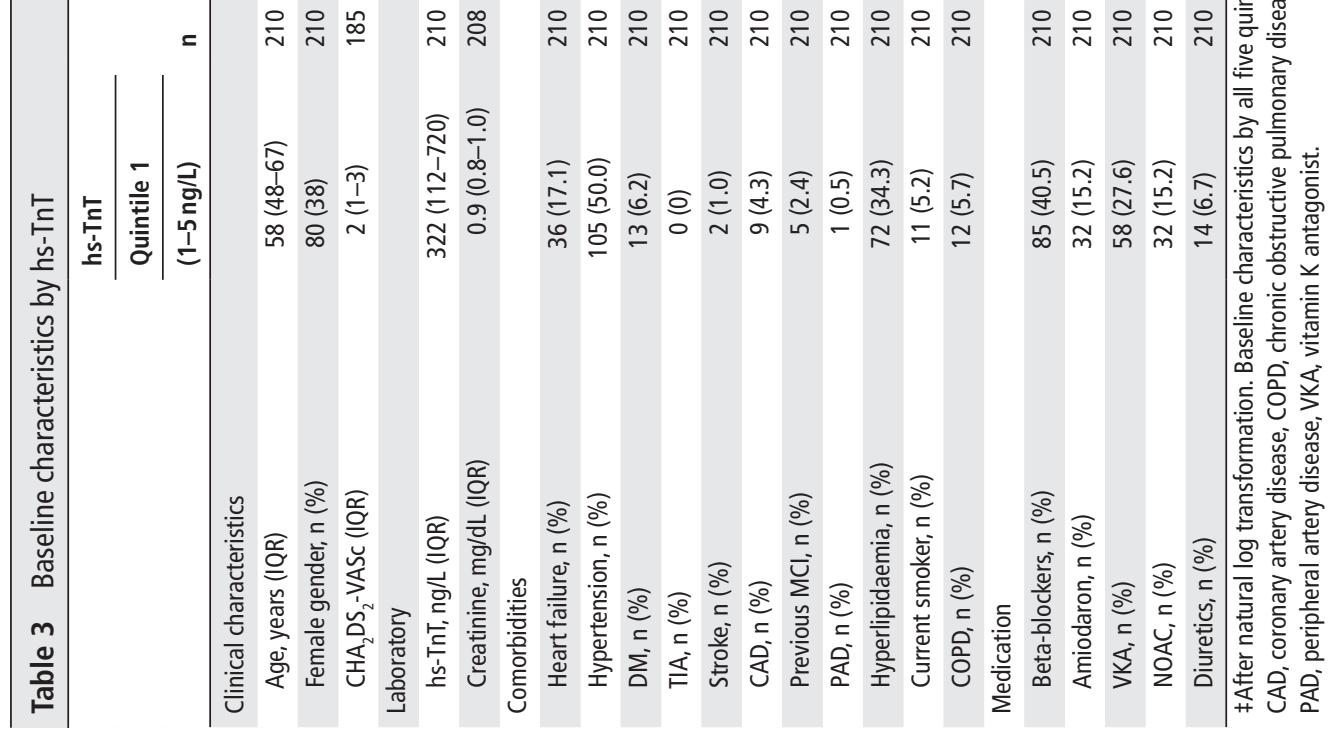




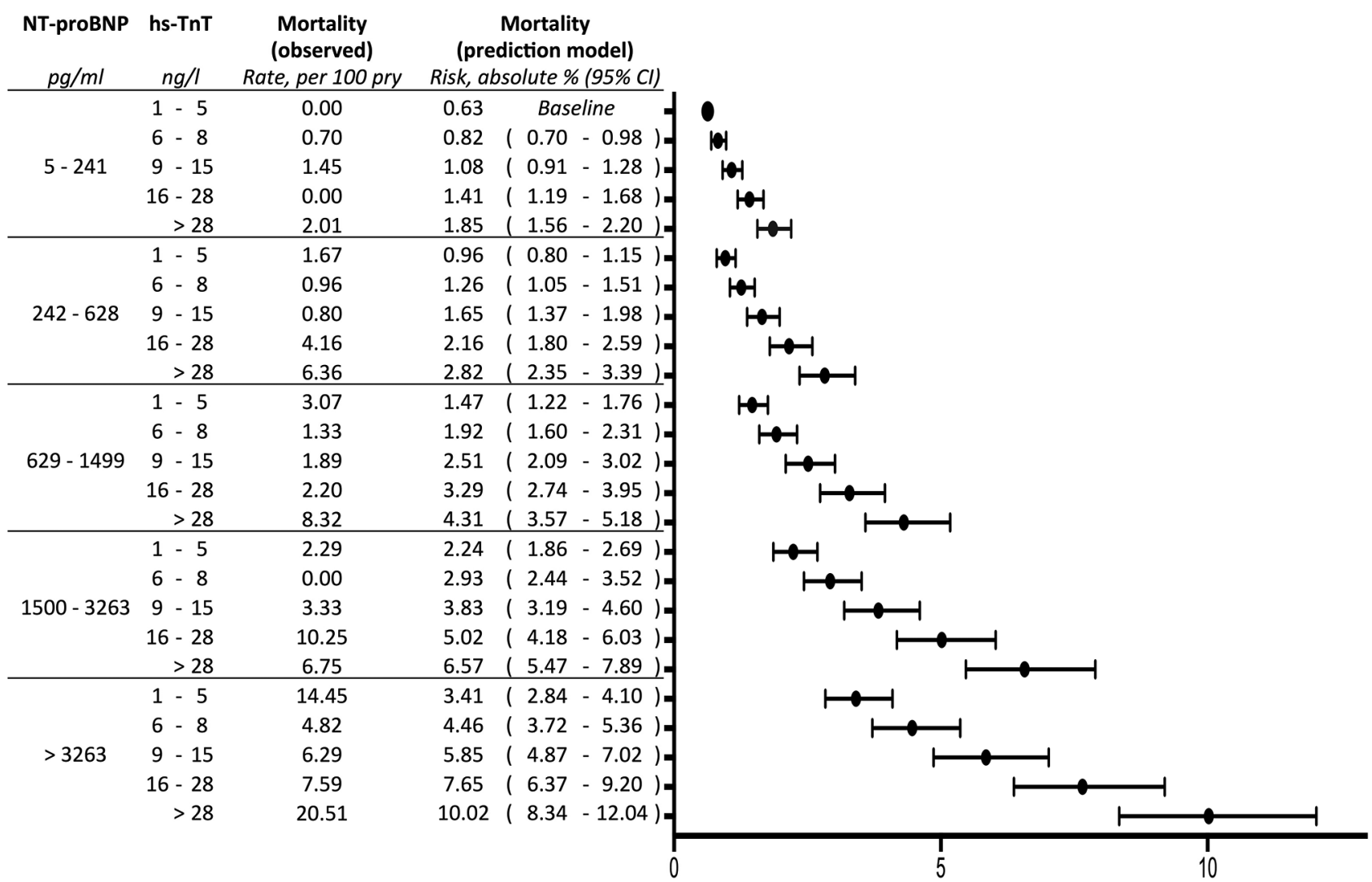

Figure 2 Mortality in patients presenting in an emergency department because of AF by NT-proBNP and hs-TnT (prediction model: Cox proportional-hazards regression; NT-proBNP and hs-TnT adjusted for the clinical risk factors age, female gender, heart failure, chronic obstructive pulmonary disease, serum creatinine, hypertension, coronary artery disease, hyperlipidaemia, diabetes mellitus, peripheral artery disease, stroke, current smoking, pulmonary vein isolation, beta-blocker therapy, diuretic therapy). AF, atrial fibrillation; NT-proBNP, N-terminal pro-brain natriuretic peptide; hs-TnT, high-sensitive troponin T.

hs-TnT quintiles. In multivariate analysis, adjusting for age, female gender, heart failure, COPD, serum creatinine, arterial hypertension, coronary artery disease, hyperlipidaemia, diabetes mellitus, peripheral artery disease, history of stroke, history of TIA, current smoking, pulmonary vein isolation, beta-blocker and diuretic therapy, hazard for death increased with every quintile of NT-proBNP by 1.53 and by 1.31 with every quintile of hs-TnT (table 3, figure 2). Adjustment for $\mathrm{CHA}_{2} \mathrm{DS}_{2}$-VASc resulted in an HR of 1.70 for each increase of NT-pro-BNP quintile and 1.52 for each increase of hs-TnT quintile for mortality (table 3).

There was no interaction between NT-pro-BNP and hs-TnT on their effect on outcome. Sensitivity analysis indicated robust estimates regarding handling of correlated data and biomarker categorisation (table 3 ).

\section{DISCUSSION}

Presentation and symptoms vary widely for patients with AF admitted to an ED; the demand for an improved risk stratification seems reasonable to facilitate optimal and cost-effective care. ${ }^{14} 15$ The present study evaluated the value of cardiac biomarkers in predicting mortality in patients and we could clearly demonstrate that both elevated NT-proBNP and hs-Troponin $\mathrm{T}$ levels at the time of admission were strongly and independently associated with increased mid-term mortality.

\section{NT-proBNP, hs-TnT and mortality in AF}

NT-proBNP and cardiac troponins have been successfully evaluated to predict prognosis in various fields of cardiovascular medicine. ${ }^{81213}$ To our best knowledge, this is the first study investigating the predictive value of both NT-proBNP and hs-TnT for all-cause mortality in patients presenting because of symptomatic AF to an ED. We found that both elevated NT-proBNP and high hs-TnT levels at the time of admission are independently associated with increased mortality in patients presenting because of $\mathrm{AF}$ in an ED. Following adjustment for clinical factors, the hazard for death increases with every quintile of NT-proBNP by 1.53 and by 1.31 with every quintile of hs-TnT.

Comparing our findings to previous reports, some specifics have to be considered: the RELY study comprised symptomatic and asymptomatic patients with rather long-lasting AF with a well-controlled median heart rate of 72 (IQR 62-82) per minute ${ }^{8}$ In contrast, in our present cohort, all patients were symptomatic, median heart rate exceeded 100 beats per minute and duration of $\mathrm{AF}$ episodes was short in general; these characteristics are typical for patients with symptomatic AF presenting to an $\mathrm{ED}$, thus fostering the robustness of our findings for application as a risk stratification tool. ${ }^{16}$ Second, Stoyanov et al have reported recently hs-TnT levels of patients with $\mathrm{AF}$ in a similar setting; in line with our observation, a direct association between symptomatic AF, biomarker levels and mortality has been observed. As this particular study did not focus primarily on symptomatic $\mathrm{AF}$ as the primary diagnosis as patients with other reasons for hs-TnT level elevations have been included, residual confounding cannot be excluded, however. ${ }^{17}$ In contrast, our study comprised unselected patients seeking help because of symptoms primary due to AF reducing this potential risk of confounding significantly. Last, we could demonstrate a clear biological gradient as 
Table 4 Baseline characteristics by biomarker availability

\begin{tabular}{|c|c|c|c|}
\hline & All episodes & $\begin{array}{l}\text { Incomplete } \\
\text { information }\end{array}$ & $\begin{array}{l}\text { Both biomarkers } \\
\text { available }\end{array}$ \\
\hline & $n=2754$ & $\mathrm{n}=974$ & $n=1780$ \\
\hline \multicolumn{4}{|l|}{ Clinical presentation } \\
\hline Age, years (IQR) & $68(58-75)$ & $67(56-75)$ & $68(59-75)$ \\
\hline Female gender, $\mathrm{n}(\%)$ & $1199(44)$ & $472(48)$ & $728(41)$ \\
\hline $\mathrm{CHA}_{2} \mathrm{DS}_{2}$-VASC (IQR) & $3(1-4)$ & $2(1-4)$ & $3(2-4)$ \\
\hline \multicolumn{4}{|l|}{ Comorbidities } \\
\hline Heart failure, n (\%) & $628(22.8)$ & $228(23.4)$ & $400(22.5)$ \\
\hline Hypertension, $\mathrm{n}(\%)$ & $1696(61.6)$ & $548(56.3)$ & $1151(64.7)$ \\
\hline $\mathrm{DM}, \mathrm{n}(\%)$ & 401 (14.6) & $120(12.3)$ & $282(15.8)$ \\
\hline TIA, n (\%) & $46(1.7)$ & $11(1.1)$ & $35(2.0)$ \\
\hline Stroke, $n(\%)$ & $141(5.1)$ & $49(5.0)$ & $92(5.2)$ \\
\hline CAD, n (\%) & $458(16.6)$ & $132(13.6)$ & $326(18.3)$ \\
\hline Previous $\mathrm{MCl}, \mathrm{n}(\%)$ & $219(8.0)$ & $56(5.7)$ & $163(9.2)$ \\
\hline PAD, $n(\%)$ & $107(3.9)$ & $25(2.6)$ & $82(4.6)$ \\
\hline Hyperlipidaemia(\%) & $840(30.5)$ & $277(28.4)$ & $565(31.7)$ \\
\hline Current smoker, n (\%) & $130(4.7)$ & $37(3.8)$ & $93(5.2)$ \\
\hline COPD, n (\%) & $256(9.3)$ & $100(10.3)$ & $156(8.8)$ \\
\hline \multicolumn{4}{|l|}{ Medication } \\
\hline Beta-blockers, n (\%) & $1133(41.1)$ & $382(39.2)$ & $751(42.2)$ \\
\hline Amiodaron) & $477(17.3)$ & $138(14.2)$ & $339(19.0)$ \\
\hline VKA, n (\%) & $766(27.8)$ & $249(25.6)$ & $517(29.0)$ \\
\hline NOAC, n (\%) & $355(12.9)$ & $120(12.3)$ & $237(13.3)$ \\
\hline Diuretics, n (\%) & $567(20.6)$ & $180(18.5)$ & $389(21.9)$ \\
\hline
\end{tabular}

CAD, coronary artery disease; COPD, chronic obstructive pulmonary disease; $D M$, diabetes mellitus; hs-TnT-sensitive troponin $\mathrm{t}, \mathrm{MCl}$, myocardial infarction;NOAC, new oral anticoagulant; NT-proBNP, N-terminal pro-brain natriuretic peptide ; $P A D$, peripheral artery disease; $\mathrm{VKA}$, vitamin $\mathrm{K}$ antagonist.

the hazard for death increases independently with every quintile of hs-TnT and NT-proBNP.

\section{Pathophysiological considerations}

Cardiac troponins have been primarily introduced as sensitive biomarkers for the inclusion and exclusion of acute myocardial infarction; however, it has been previously shown that elevation of cardiac troponins might also indicate the severity of other non-cardiac-related conditions; as symptomatic AF is an abnormal physiological state, elevated levels of troponins might reflect an oxygen demand/delivery mismatch and changes in microvascular blood flow thus indicating the urgency or emergency of disease. ${ }^{18-21}$ This hypothesis might further be strengthened by the fact that BNPs are mainly released in response to high wall tension during states of haemodynamic stress. ${ }^{22}$

\section{Clinical implications}

EDs play a key role in the management of the inhomogeneous group of patients with $\mathrm{AF}^{32324}$; the use of risk stratification tools is mandatory for a time-effective and cost-effective treatment and easily accessible biomarkers might assist in this goal-oriented and risk-oriented approach. ${ }^{8}{ }^{13}$ As to our observations, NT-proBNP and hs-TnT may serve as those ideal biomarkers for identification of patients with symptomatic $\mathrm{AF}$ at risk; due to the increased risk of all-cause mortality, an extensive screening and more aggressive treatment stratified should be considered in all patients with AF with elevated NT-proBNP or/and hs-TnT levels presenting to an ED.

\section{Strengths and limitations}

The present study inherits all known limitations of a prospective observational study design that have to be acknowledged; those limitations are not limited to individual patient profiles, management at the ED and in further care only. However, this study covers a large cohort of consecutive patients within a limited and short time frame that decreases the potential negative impact on our conclusions. Due to the nature of an ED setting, only 1780 of a total 2574 episodes were available for analysis as to missing biomarker data; however, baseline characteristics between those included and excluded showed no significant differences lowering the potential impact of this limitation (table 4). Besides the clear demonstration of a biological gradient between biomarker levels and mid-term mortality, the probably greatest strength of our study is all-cause mortality being the primary outcome measure; as the issuance of death certificates is mandatory in Austria and registered by a central, state-controlled office, information bias can be nearly excluded. Due to our single-centre design, however, our results will not be fully generalisable to other settings and populations; our results ideally need confirmation in larger populations and this might allow then to estimate conceivable cut-off values for risk estimation and stratification that we were not able to due to sample size limitations.

\section{CONCLUSION}

Indicating substantially increased mid-term mortality in patients with symptomatic AF, NT-proBNP and hs-TnT could help deciding whether hospitalisation and/or specific long-term management are expedient in high-risk patients. Due to the

\section{Key messages}

What was already known on this subject?

- It is common knowledge that atrial fibrillation (AF) is regarded as a risk factor for increased mortality but information on specific patient subgroups at risk is scarce. $\mathrm{N}$-terminal pro-brain natriunatriureticde (NT-proBNP) and cardiactroponins are known, powerful predictors of prognosis in various fields of cardiovascular medicine. It was recently proposed that cardiac troponins and NT-proBNP improve existing risk stratification in patients with AF.

\section{What might this study add?}

- Patients with symptomatic AF are at a substantial risk for increased mortality (4.72/100 pry) during follow-up. Elevated NT-proBNP and high-sensitive troponin T (hs-TnT) levels are capable to predict mid-term outcome independently: following adjustment for established risk factors, hazards for death increases with every quintile of NT-proBNP by 1.53 ( $\mathrm{HR} ; 95 \% \mathrm{Cl} 1.27$ to $1.83 ; \mathrm{p}<0.001)$ and by $1.31(\mathrm{HR} ; 95 \% \mathrm{Cl}$ 1.10 to $1.55 ; p=0.002$ ) with every quintile of hs-TnT).

\section{How might this impact on clinical practice?}

- Indicating substantially increased mid-term mortality in patients with symptomatic AF, NT-proBNP and hsTnT could help to decide whether hospitalisation and/or specific long-term management are expedient in high-risk patients. Due to the increased risk of all-cause mortality, an extensive screening and more aggressive treatment stratified by elevated NT-proBNP and hs-TnT might be considered in patients with AF presenting to an emergency department. 
increased risk of all-cause mortality, an extensive screening and more aggressive treatment stratified by elevated NT-proBNP or/ and hs-TnT might be considered in AF-patients presenting to an ED.

Acknowledgements The authors are grateful to Ms Mikulasek and Mr Kupez, as well as llinca Damian for supporting our study.

Funding The authors have not declared a specific grant for this research from any funding agency in the public, commercial or not-for-profit sectors.

Competing interests None declared.

Ethics approval This academic trial (registered at ; Unique identifier: NCT03272620) was approved by Ethics Committee of the Medical University of Vienna, Vienna General Hospital (EK-No. 1568/2014) and written consent was mandatory prior inclusion

Provenance and peer review Not commissioned; externally peer reviewed.

Open access This is an open access article distributed in accordance with the Creative Commons Attribution Non Commercial (CC BY-NC 4.0) license, which permits others to distribute, remix, adapt, build upon this work non-commercially, and license their derivative works on different terms, provided the original work is properly cited, appropriate credit is given, any changes made indicated, and the use is non-commercial. See: http://creativecommons.org/licenses/by-nc/4.0/.

\section{REFERENCES}

1 Russo V, Navarin S, Zampini G, et al. Management of atrial fibrillation in the Emergency Department: current approach and future expectations. Eur Rev Med Pharmacol Sci 2013:17:3132-47.

2 Gallagher $C$, Elliott AD, Wong CX, et al. Integrated care in atrial fibrillation: a systematic review and meta-analysis. Heart 2017;103.

3 Kirchhof P, Benussi S, Kotecha D, et al. 2016 ESC Guidelines for the management of atrial fibrillation developed in collaboration with EACTS. Eur Heart J 2016 37:2893-962.

4 Afilal M, Yalaoui F, Dugardin F, et al. Forecasting the Emergency Department Patients Flow. J Med Syst 2016;40:175

5 Fonarow GC, Peacock WF, Phillips CO, et al. Admission B-type natriuretic peptide levels and in-hospital mortality in acute decompensated heart failure. J Am Coll Cardiol 2007;49:1943-50.

6 Kragelund C, Grønning B, Køber L, et al. N-terminal pro-B-type natriuretic peptide and long-term mortality in stable coronary heart disease. N Engl J Med 2005;352:666-75.

7 Hijazi Z, Wallentin L, Siegbahn A, et al. N-terminal pro-B-type natriuretic peptide for risk assessment in patients with atrial fibrillation: insights from the ARISTOTLE Trial (Apixaban for the Prevention of Stroke in Subjects With Atrial Fibrillation). J Am Coll Cardiol 2013;61:2274-84.
8 Hijazi Z, Oldgren J, Andersson U, et al. Cardiac biomarkers are associated with an increased risk of stroke and death in patients with atrial fibrillation: a Randomized Evaluation of Long-term Anticoagulation Therapy (RE-LY) substudy. Circulation 2012:125:1605-16.

9 Omland T, de Lemos JA, Sabatine MS, et al. A sensitive cardiac troponin T assay in stable coronary artery disease. N Engl J Med 2009:361:2538-47.

10 Antman EM, Tanasijevic MJ, Thompson B, et al. Cardiac-specific troponin I levels to predict the risk of mortality in patients with acute coronary syndromes. $N$ Engl J Med 1996;335:1342-9.

11 Resl M, Clodi M, Vila G, et al. Targeted multiple biomarker approach in predicting cardiovascular events in patients with diabetes. Heart 2016;102:1963-8.

12 Naffaa ME, Nasser R, Manassa E, et al. Cardiac troponin-I as a predictor of mortality in patients with first episode acute atrial fibrillation. QJM 2017;110:507-11.

13 Hijazi Z, Oldgren J, Siegbahn A, et al. Biomarkers in atrial fibrillation: a clinical review. Eur Heart J 2013:34:1475-80.

14 Flaker GC, Belew K, Beckman K, et al. Asymptomatic atrial fibrillation: demographic features and prognostic information from the Atrial Fibrillation Follow-up Investigation of Rhythm Management (AFFIRM) study. Am Heart J 2005:149:657-63.

15 Hagens VE, Ranchor AV, Van Sonderen E, et al. Effect of rate or rhythm control on quality of life in persistent atrial fibrillation. Results from the Rate Control Versus Electrical Cardioversion (RACE) Study. J Am Coll Cardiol 2004;43:241-7.

16 Stiell IG, Clement CM, Rowe BH, et al. Outcomes for emergency department patients with recent-onset atrial fibrillation and flutter treated in canadian hospitals. Ann Emerg Med 2017:69:562-71.

17 Stoyanov KM, Giannitsis E, Biener M, et al. Prognostic value of elevated highsensitivity cardiac troponin $\mathrm{T}$ in patients admitted to an emergency department with atrial fibrillation. Europace 2018:20:582-8.

18 Eggers KM, Lind L, Ahlström H, et al. Prevalence and pathophysiological mechanisms of elevated cardiac troponin I levels in a population-based sample of elderly subjects. Eur Heart J 2008;29:2252-8

19 Neukamm A, Einvik G, Didrik Høiseth A, et al. The prognostic value of measurement of high-sensitive cardiac troponin $T$ for mortality in a cohort of stable chronic obstructive pulmonary disease patients. BMC Pulm Med 2016;16:164

20 Ralli S, Horwich TB, Fonarow GC. Relationship between anemia, cardiac troponin I, and B-type natriuretic peptide levels and mortality in patients with advanced heart failure. Am Heart J 2005:150:1220-7.

21 Ostermann M, Ayis S, Tuddenham E, et al. Cardiac troponin release is associated with biomarkers of inflammation and ventricular dilatation during critical illness. Shock 2017:47:702-8.

22 Daniels LB, Maisel AS. Natriuretic peptides. J Am Coll Cardiol 2007:50:2357-68.

23 Andrade J, Khairy P, Dobrev D, et al. The clinical profile and pathophysiology of atrial fibrillation: relationships among clinical features, epidemiology, and mechanisms. Circ Res 2014;114:1453-68.

24 Kim D, Yang PS, Jang E, et al. Increasing trends in hospital care burden of atrial fibrillation in Korea, 2006 through 2015. Heart 2018;104:2010-7. 